\title{
Different slopes for different folks: Process analysis of spatial aptitude
}

\author{
RANDALL J. MUMAW \\ University of Pittsburgh, Pittsburgh, Pennsylvania \\ JAMES W. PELLEGRINO \\ University of California, Santa Barbara, California \\ ROBERT V. KAIL, JR. \\ Purdue University, West Lafayette, Indiana \\ and \\ PHILIP CARTER \\ University of Pittsburgh, Pittsburgh, Pennsylvania
}

\begin{abstract}
Adults differing in spatial ability were tested on problems involving the mental rotation of familiar alphanumeric stimuli and unfamiliar stimuli drawn from the Primary Mental Abilities Space Test. Individual differences in spatial ability were associated with speed rather than accuracy of mental rotation processes. Ability differences were correlated with the time to rotate familiar alphanumerics and the additional time to encode, compare, and rotate unfamiliar stimuli. The results are discussed in terms of differences in elementary information processes associated with the representation and transformation of visual information.
\end{abstract}

Information processing models have been applied successfully to the analysis of individual differences in cognitive abilities. The majority of such research has focused on performance in verbal processing tasks (e.g., Fredericksen, 1980; Hunt, 1980), as well as on inductive and deductive reasoning tasks (e.g., Pellegrino \& Glaser, 1980, 1982; Sternberg, 1977, 1979). Spatial aptitude represents another major individual differences factor, and information processing models have been developed for spatial tasks such as mental rotation (Cooper \& Shepard, 1973). However, such models have not been used extensively to examine the components of processing that contribute to individual differences in spatial ability (see, however, Cooper, 1980, and Egan, 1979).

The present research focused on individual differences in performance for a simple spatial relations task-the Primary Mental Abilities (PMA) Space Test (Thurstone, 1958). This test was chosen for two reasons. First, the PMA is prototypical of many measures of spatial aptitude in which an individual must "mentally rotate" a twodimensional stimulus in the picture plane in order to differentiate it from other similar stimuli and match it against some standard. Second, the PMA loads heavily on the spatial relations factor in factor analytic studies of the structure of intelligence (Cattell, 1971; Lohman, 1979; Smith, 1964; Thurstone, 1938). Figure 1 shows a typi-

Requests for reprints should be addressed to the second author, Department of Education, University of California at Santa Barbara, Santa Barbara, CA 93106. cal item from the PMA. A referent stimulus is on the left, and five comparison stimuli are on the right. A decision must be made about the match between the referent and each comparison stimulus given rotation in the picture plane. Mismatches involve rotation plus mirror-image reversals. The PMA consists of 30 problems of the type illustrated in Figure 1. Only $7 \mathrm{~min}$ is provided for problem solution, which averages to less than $3 \mathrm{sec}$ per comparison to complete the test. Most individuals fail to complete all problems in the time allotted, and individual differences are primarily a function of the number of problems solved, rather than of overt errors, that is, failures to detect matches and mismatches.

Solution of simple spatial relations problems such as those found on the PMA can be related to the general process model proposed by Cooper and Shepard (1973) for mental rotation tasks. The process model was based upon data obtained in a paradigm that required individuals to decide, as rapidly as possible, whether or not two stimuli presented in different visual orientations were the same. This single-trial comparison of a stimulus pair closely resembles the individual comparisons that must be made to solve PMA problems. An example of the application of this paradigm is a study by Cooper (1975), in which she presented two nonsense shapes that differed in orientation from $0^{\circ}$ to $300^{\circ}$. Subjects judged whether the shapes were identical or mirror images of one another. Response latencies in this task were a linearly increasing function of the difference in orientation (angular disparity) between the two shapes. Such results have heen inter- 

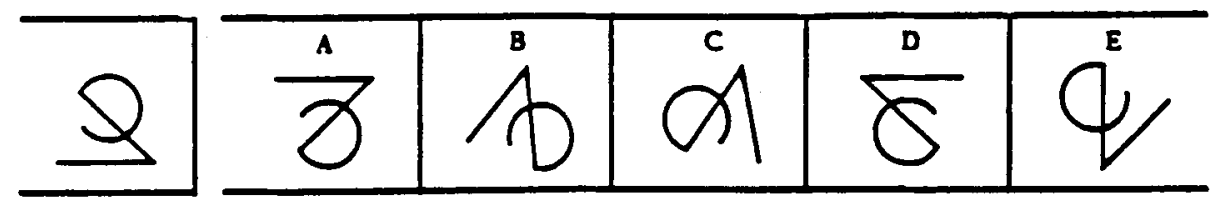

Figure 1. Example item from the PMA Space Test.

preted as indicating that subjects mentally rotate the stimuli in a manner analogous to the actual physical rotation of the object. The greater the "mental distance" to be traveled, the longer it takes to solve the problem.

Cooper and Shepard (1973) presented evidence that response latency on these problems reflects four discrete stages of processing. The first stage of processing requires encoding of the stimuli. Encoding involves representing the stimuli, that is, their identities and orientations, and storing this information in working memory. The second phase of processing involves rotation of the mental representation of the nonvertical stimulus to bring it into congruence with the vertical stimulus. This phase is followed by a comparison of the stimulus representations to determine whether or not they are identical. The outcome of the comparison leads to a positive or negative response. Only the second stage of processing (i.e., mental rotation) is affected by the orientation of the stimulus (Cooper \& Shepard, 1973). Encoding, comparing, and responding take approximately the same amount of time, regardless of the orientation of the stimulus. Consequently, the overall equation for reaction time (RT) in this task is generally written as $R T=x(r)+(e+c+m)$, where $x$ represents the angular disparity between the stimuli being compared and $r, e, c$, and $m$ represent the times for rotation, encoding, comparison, and motor response, respectively. The slope of the function relating RT to angular disparity is used to estimate the rate of mental rotation, whereas the intercept provides an estimate of the total time necessary for the remaining processes that are presumed constant over problems.

The process analysis of performance on a mental rotation problem provides a scheme for the analysis of individual differences in simple spatial relations performance. If the processes involved in solving mental rotation problems can be reliably estimated for individuals, then what remains to be determined are the respective contributions of these processes to skill differences as measued by a reference test such as the PMA.

Individual differences in performance on a speeded test such as the PMA may be due primarily to speed differences in the cognitive process of mental rotation. To estimate this process in its simplest form, the slope value for the rotation of familiar stimuli such as alphanumerics can be determined. The intercept of the function for processing alphanumeric stimuli is an estimate of the time to encode, compare, and respond to familiar stimuli, and it, too, may be related to individual differences in reference-test performance. A potentially important aspect of performance on a test such as the PMA may involve the capacity to encode, compare, and rotate stimuli that are not familiar and that lack representations and labels in permanent memory. Previous research has shown that the slope for processing unfamiliar stimuli such as PMA characters is greater than the slope for processing familiar alphanumerics (e.g., Kail, Pellegrino, \& Carter, 1980). Similarly, there is a higher intercept for processing unfamiliar stimuli of the PMA type. Thus, it is necessary to consider the additional times associated with encoding, comparing, and rotating unfamiliar stimuli as potentially important aspects of skill differences on a reference test such as the PMA. All of these aspects of processing, as well as accuracy, were considered in the present study.

\section{METHOD}

\section{Design and Materials}

A three-factor within-subjects design, representing two stimulus types (alphanumerics vs. PMA characters), six rotation values $\left(0^{\circ}, 30^{\circ}, 60^{\circ}\right.$, $90^{\circ}, 120^{\circ}$, and $\left.150^{\circ}\right)^{1}$ and two judgment types (same vs. different), was used. The alphanumeric stimuli were $4,5, F, G, J, L, P$, and $R$, which were selected because of their physical asymmetry. The PMA stimuli represented eight unfamiliar characters selected from the original test; they are shown in Figure 2. Twelve slides were prepared for each stimulus, six representing same judgments and six representing different judgments. For the same-judgmént slides, the standard and comparison stimuli were identical but differed in orientation in the picture plane by $0^{\circ}-150^{\circ}$ in six steps. For the different-judgment slides, the standard and comparison stimuli were mirror images that also differed in orientation by $0^{\circ}-150^{\circ}$ in six steps. Thus, 192 unique stimulus pairs were generated. The 192 stimulus pairs were organized into four blocks of 48 trials. Within each block, slides were ordered randomly, with the following constraints: (1) the same rotation value could not occur on consecutive trials, (2) at least 2 trials separated the occurrence of the same alphanumeric or PMA character, and (3) there was an equal occurrence of same- and different-judgment trials for each stimulus type. A Latin-square counterbalancing procedure was used for presenting trial blocks to individual subjects.

There were two separate subject groups that differed with respect to the above design. One set of subjects $(N=48)$ received two replirations of the complete design, which yielded a total of 384 trials. The second group of subjects $(\mathrm{N}=51)$ was tested on a subset of the 192-item stimulus set. This subset included the complete set of same-judgment trials (96) and half the set of different-judgment trials (48). The 48 different-judgment trials represented both stimulus types and all rotation values with a partial sampling of the eight alphanumeric and PMA characters. The second group of subjects received two replications of this partial stimulus set, which yielded a total of 288 trials. The two subject groups were tested at different times and represented separate experimental groups. However, because no significant differences between the two groups were obtained on any of the performance measures, they are treated as a single combined group for purposes of data presentation and analysis.

\section{Procedure}

Prior to being tested on the laboratory task, all subjects were administered the PMA Space Test in its standard timed testing format. The subjects were then given instructions for the laboratory task; the 

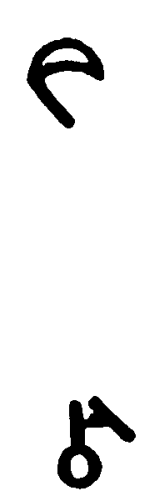
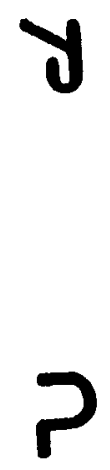

$\mathbf{G}$

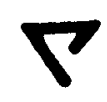

$\nabla$

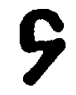

Figure 2. PMA stimuli used for experimental items.

instructions emphasized that individuals were to respond as rapidly as possible while minimizing errors. Several identical and mirror-image pairs were presented to demonstrate the distinction between same and different judgments. A series of timed practice trials was then given prior to presentation of the experimental items. On each practice and experimental trial, a slide was rear-projected onto a $17.5 \times 13 \mathrm{~cm}$ screen located approximately $1 \mathrm{~m}$ from the subject. When projected, the two alphanumeric or PMA characters were approximately $2 \mathrm{~cm}$ in length and width and $7.5 \mathrm{~cm}$ apart. Presentation of each slide activated a photocell that initiated timing (in milliseconds) by a Hunter Klockcounter. The subject's response stopped the timer and terminated presentation of the stimulus. The subject responded by pressing, with the index finger of the preferred hand, one of two response buttons labeled "same" and "different." The buttons were located on a response panel in front of the subject and equidistant from a center spot where the subject rested his/her finger.

\section{Subjects}

The subjects were 99 University of Pittsburgh undergraduates. They were either paid for their participation or given hourly credit in fulfillment of a course requirement. The 99 subjects included 53 females and 46 males.

\section{RESULTS AND DISCUSSION}

The PMA scores indicated that all levels of spatial ability were represented in the subject sample. The mean PMA score was 40.8 , with a standard deviation of $15.3 .^{2}$ The lowest score was 3 , and the highest was a single perfect score of 70 . Table 1 provides data on the distribution of raw scores and their corresponding normative percentile ranges. As shown in Table 1, the majority of subjects were in the 58th to 99 th percentiles. This is typical of adult samples drawn from a college population. A comparison between males and females yielded a significant sex difference $[\mathrm{t}(95)=2.28, \mathrm{p}<.05]$, with means of 44.5 and 37.6 for males and females, respectively. Sex

Table 1

PMA Score Distribution

\begin{tabular}{ccc}
\hline Raw Score Interval & Frequency & Percentile Range \\
\hline $0-10$ & 2 & $5-19$ \\
$11-20$ & 5 & $19-31$ \\
$21-30$ & 11 & $33-55$ \\
$31-40$ & 25 & $58-81$ \\
$41-50$ & 27 & $83-95$ \\
$51-60$ & 21 & $96-99$ \\
$61-70$ & 6 & $99+$ \\
\hline
\end{tabular}

differences are common on spatial relations tests such as the PMA (McGee, 1979; Thurstone, 1958). However, regression analyses showed that variance attributable to sex was negligible relative to other systematic individual difference variance within the entire sample.

For each subject, RT means were computed on the basis of the 16 separate same-judgment trials for each rotation value and stimulus type. ${ }^{3}$ Only correct response latencies were used in the computation of the individual subject means. Error rates were low, with an average error probability of .03 for alphanumerics and .09 for PMA stimuli. Figure 3 shows the group mean data and the least squares regression lines. As shown in Figure 3, the group mean data for both stimulus types were highly linear, with $r^{2}$ values above .95 . The PMA stimuli produced higher average intercepts and steeper slope values consistent with previous research (e.g., Kail et al., 1980).

Linear functions were also fit to the individual subject data for both stimulus types. For both alphanumeric and PMA stimuli, the $r$ values were significant at the $p<.05$ level $(r \geq .811)$ for all but nine subjects. Of these nine subjects, eight had only one nonsignificant $r$ value, and in all cases the $r$ was greater than .70. The mean $r$ values for alphanumeric and PMA stimuli were .92 and .94 , respectively. The uniformly high linear fits for individual subjects led to nonsignificant correlations between measures of linear fit $\left(r^{2}\right)$ and PMA scores.

Table 2 contains mean, standard deviation, and minimum and maximum score data for slopes and intercepts. As can be seen in Table 2, there were substantial individual differences in each measure of performance. Also shown in Table 2 are individual subject overall error probabilities indicating the high average accuracy levels. The standard deviation reflects a substantial skewness in the distribution, with $80 \%$ of the subjects having an error rate of less than an $8 \%$ and the remaining $20 \%$ having error rates ranging from $9 \%$ to $27 \%$.

An important issue relative to the individual subject parameter estimates is their reliability. Table 3 shows both the pattern of intercorrelations among the latency parameters and their individual split-half reliabilities (along the diagonal). All four parameters had reliabilities above .82. As might be expected, the highest correlations are between measures assumed to share some 


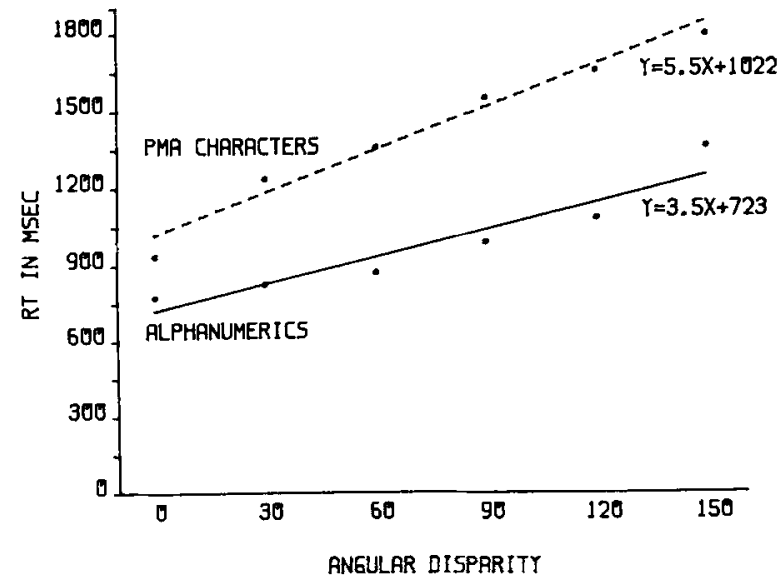

Figure 3. Mental rotation mean latency data as a function of stimulus type and angular disparity.

commonality of processing operations. For example, the intercept for alphanumeric stimuli is highly correlated with the intercept for PMA stimuli, and there is less shared variance between slope and intercept measures. ${ }^{4}$ The correlational pattern thus provides evidence of convergent and discriminant validity for the various process measures. The average correlation between corresponding process measures was .75 , whereas the average correlation between noncorresponding process measures was .30 . However, the correlations are not of the level at which it can be assumed that the two slope measures or the two intercept measures assess completely identical sources of variance.

Figure 4 shows mean values for each of the four process measures for subjects representing four levels of ability defined by PMA test performance. The four groups represent subjects with percentile ranks of 5-55 $(\mathrm{N}=18)$, 58-81 $(\mathrm{N}=25), 83-95(\mathrm{~N}=27)$, and $96-99+(\mathrm{N}=27)$. The left panel of Figure 4 shows data for the two intercept measures. As can be seen in Figure 4, ability differences were not substantial for the alphanumeric intercept, with a difference of $94 \mathrm{msec}$ between the subjects of lowest and highest ability. In contrast, a substantial ability effect was obtained for the PMA character intercept, with a difference of $291 \mathrm{msec}$ between the subjects of lowest and highest ability. The right panel of Figure 4 shows the data for the slope measures. Ability differences were obtained for both slope measures. However, the absolute difference between the subjects of lowest and highest ability was larger for the PMA stimuli. The low- high group differences were 4.04 and $2.79 \mathrm{msec} /{ }^{\circ}$ for PMA and alphanumeric stimuli, respectively.

The group differences shown in Figure 4 were supported by simple correlations between the four latency measures and PMA scores. The last column of Table 3 contains the simple $r$ values. Both slope measures yielded similar significant correlations with reference-test performance. The two intercept measures yielded correlations of different magnitude, with the PMA character intercept more highly correlated with PMA scores. A multiple regression analysis using these four latency measures proved unsatisfactory for two reasons. First, the substantial variance shared by the two slope and two intercept measures led to a final solution in which only the PMA intercept and slope measures were significant predictors of PMA scores. Second, such an analysis does not help to separate out unique variance attributable to processing familiar versus unfamiliar stimuli.

In the case of the two slope measures, it can be hypothesized that correlations involving the PMA slope reflect variance due to the speed of a basic rotation process and variance due to the additional time required to rotate unfamiliar stimuli. Separating out these two sources of variance should yield a multiple $R$ that exceeds the simple $r$ yielded by the PMA slope measure alone. When this is done, $24 \%$ of the variance in PMA test scores is accounted for, in contrast to $19 \%$ when only the PMA slope measure is used. In addition, both sources of variance are significant contributors in the multiple regression (ps < .05). A similar argument can be made for correlations involving the PMA intercept measure. Separating out variance attributable to basic encoding, comparison, and motor response processes, and variance attributable to the additional time required to encode and compare unfamiliar stimuli yields a multiple $\mathrm{R}$ accounting for $21 \%$ of the variance in PMA test scores, in contrast to the $18 \%$ when only the PMA intercept measure is used. In this case, however, the only significant source of variance in the multiple regression is attributable to the additional time to encode and compare unfamiliar stimuli. The other source of variance is only marginally significant $(p \cong .10)$.

The two slope and intercept measures were therefore used to derive four parameters that formed the basis of an alternative multiple regression analysis of individual differences. The four parameters were: (1) the time to encode, compare, and respond to familiar stimuli, alphanumeric intercept; (2) the additional time for encoding and

Table 2

Means, Standard Deviations, and Range for Individual Subject Performance Measures

\begin{tabular}{lcccc}
\multicolumn{1}{c}{ Measure } & Mean & SD & Minimum & Maximum \\
\hline PMA Slope $\left(\mathrm{msec} /{ }^{\circ}\right.$ ) & 5.50 & 3.68 & 1.27 & 23.06 \\
Alphanumeric Slope $\left(\mathrm{msec} /{ }^{\circ}\right.$ ) & 3.49 & 2.61 & .55 & 20.29 \\
PMA Intercept (msec) & 1022 & 240 & 676 & 2109 \\
Alphanumeric Intercept (msec) & 723 & 157 & 468 & 1299 \\
Error Probability & .06 & .05 & .00 & .27 \\
\hline
\end{tabular}




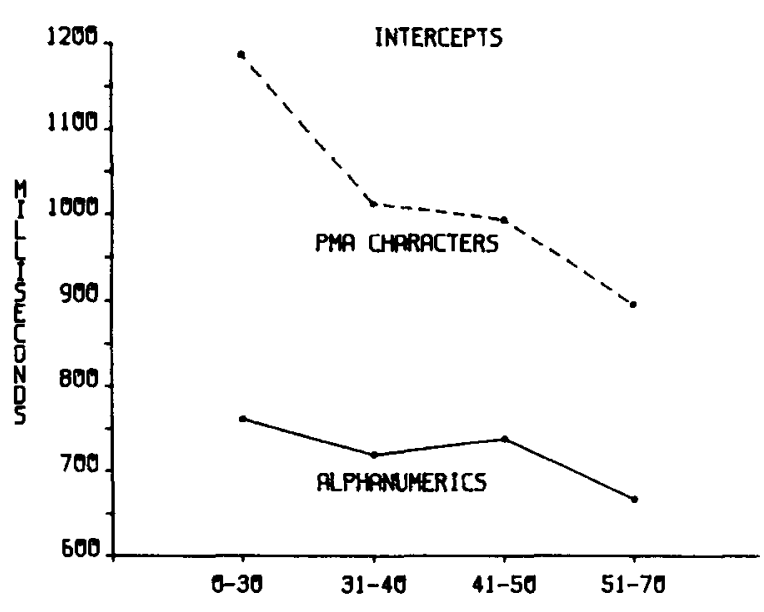

PMA TEST SCORE RANGES

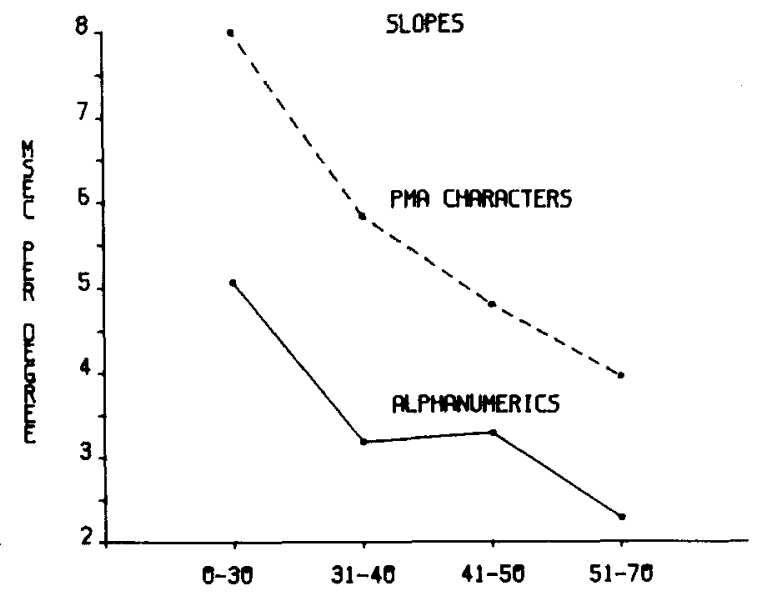

PMA TEST SCORE RANGES

Figure 4. Mean values for slope and intercept measures as a function of PMA ability level.

comparing unfamiliar PMA stimuli, intercept difference; (3) the time to rotate familiar stimuli, alphanumeric slope; and (4) the additional time for rotating unfamiliar PMA stimuli, slope difference. The multiple regression results with PMA scores as the criterion $(R=.57)$ are shown in Table 4. Table 4 shows that individual differences in PMA test performance are primarily a function of (1) the speed of the mental rotation process for familiar stimuli, (2) the additional time to rotate unfamiliar PMA stimuli, and (3) the additional time associated with encoding and comparing unfamiliar PMA stimuli.

Two additional negative results are of importance. First, both the simple and the multiple regression analyses showed that the least important latency parameter for predicting performance was the intercept for processing alphanumeric stimuli. Such a result is of interest because this parameter accounts for approximately $50 \%$ of the total time required to solve a typical rotation item. Second, the number of errors is generally low on both PMA and alphanumeric rotation problems, and individual differences in error rates on experimental items are not related to skill differences on the PMA. The actual value for the correlation between errors and PMA scores was $r=-.18$ $(p>.05)$. Thus, speed rather than accuracy differences seem to account for spatial aptitude differences as measured by the PMA, a simple spatial relations task. The particular speed differences that partially account for individual differences on the PMA spatial relations test involve a basic mental rotation process and the speed of encoding, comparing, and rotating unfamiliar stimuli.
This is consistent with Lohman's (1979) analysis of differences between spatial relations and more complex spatial visualization tasks. The latter seem to assess both speed and power differences among individuals.

\section{GENERAL DISCUSSION}

The simple and multiple regression analyses of individual subject data suggest some of the important processing components contributing to overall individual differences in performance on spatial relations tasks such as the PMA. Several issues remain to be addressed with respect to the group and individual differences that were found, particularly with regard to the encoding, comparison, and rotation of the unfamiliar PMA stimuli. One issue is that of why such stimuli take longer to rotate and show greater variability with respect to the duration of the rotation process. Related to this is the question of why some individuals have such difficulty in processing these stimuli.

One hypothesis is that, for unfamiliar stimuli, and perhaps also for familiar stimuli, rotation may be a composite of several processes, rather than a holistic process as postulated by some (e.g., Shepard, 1975). The solution of even a simple mental rotation problem may involve repetitive processing of a series of separate stimulus elements. Such an assumption can begin to explain why alphanumerics, which may be holistically processed given the availability of a long-term memory representation, are rotated more rapidly than PMA stimuli. The latter may

Table 3

Parameter Correlations and Reliabilities

\begin{tabular}{lccccc}
\hline & Alphanumeric Intercept & PMA Intercept & Alphanumeric Slope & PMA Slope & PMA Test Score \\
\hline Alphanumeric Intercept & $.82^{* * *}$ & $.77^{* * *}$ & $.22^{*}$ & .14 & $-.24^{*}$ \\
PMA Intercept & $(.93)$ & $.84^{* * *}$ & $.52^{* * *}$ & $.32^{* *}$ & $-.43^{* *}$ \\
Alphanumeric Slope & $(.26)$ & $(.61)$ & $.87^{* * *}$ & $.72^{* * *}$ & $-.42^{* *}$ \\
PMA Slope & $(.17)$ & $(.39)$ & $(.85)$ & $.82^{* * *}$ & $-.44^{* *}$ \\
\hline
\end{tabular}

Note-Values shown in parentheses are corrected for attenuation.

${ }^{*} p<.05 .{ }^{* *} p<.01 .{ }^{* * *} p<.001$. 
Table 4

Multiple Regression Results with PMA Test Scores as the Criterion

\begin{tabular}{lll}
\multicolumn{1}{c}{ Predictor } & \multicolumn{1}{c}{ F } & \multicolumn{1}{c}{$\beta$} \\
\hline Error Probability & 2.37 & -.15 \\
Alphanumeric Intercept & 2.38 & -.15 \\
Intercept Difference & $4.90^{*}$ & -.24 \\
Alphanumeric Slope & $5.11^{*}$ & -.26 \\
Slope Difference & $4.67^{*}$ & -.20 \\
\hline
\end{tabular}

${ }^{*} p<.05$.

often require stimulus fractionation prior to rotation (see, e.g., Pylyshyn, 1979). Individuals who show particularly long latencies for rotating such stimuli may be forced to execute a rotation process several times because of their inability to achieve a sufficiently stable internal representation that can be holistically operated on (see, e.g., Just \& Carpenter, 1976). Similarly, the additional time to encode and compare unfamiliar PMA stimuli may be due to the need to execute a comparison process for each separate stimulus element that is rotated. The suggestion is that individual differences in spatial ability may emanate from representational and "visual memory" skills that are fundamental and that affect the total time and course of item processing. It must be noted, however, that there are substantial individual differences in the time required to rotate even highly familiar stimuli. This elementary process difference appears to be compounded when processing of unfamiliar stimuli is required.

As noted in the introduction, studies of individual differences in mental rotation parameters have been limited. Egan (1978) examined the relationship between reference abilities and mental rotation parameters for complex threedimensional stimuli of the type used by Metzler and Shepard (1974). His data indicated that both speed and accuracy measures from the mental rotation task were correlated with reference-test scores. However, the speed measure that was most highly correlated with test scores was the intercept, rather than the slope. Differences between Egan's data and the present data can be attributed to several factors. First, the mental rotation task used by Egan is considerably more difficult than the present twodimensional rotation task. Thus, it would be expected that individual differences would be associated with both speed and accuracy of processing. Pellegrino and Mumaw (1980) showed this to be the case for three-dimensional mental rotation. Second, the slope parameter obtained by Egan had a relatively low reliability. Research reported by Lansman (1981) and Pellegrino and Mumaw (1980) has shown that when more reliable process parameters are obtained for individual subjects, both slope and intercept parameters are correlated with spatial ability differences. The third possibility relates to possible range restrictions in Egan's subject sample, since all his subjects were Naval Flight Officer candidates.

When Egan's results are considered together with the present results and those reported by Lansman (1981) and Pellegrino and Mumaw (1980), a more consistent set of conclusions emerges. High-ability individuals are faster at rotating unfamiliar two- and three-dimensional stimuli, and they are also faster at encoding and comparing such stimuli. As stimulus complexity increases, there is also a higher level of accuracy exhibited by high-ability individuals. As noted earlier, these results may be attributable to representational processes. High-ability individuals may be able to create more accurate internal representations of unfamiliar stimuli and maintain the quality of such representations during spatial transformation such as rotation. More precise representations may also permit faster or more efficient execution of subsequent mental operations such as rotation and comparison.

\section{REFERENCES}

Cattell, R. B. (1971). Abilities: Their structure, growth and action. Boston: Houghton Mifflin.

COOPER, L. A. (1975). Mental transformations of random twodimensional shapes. Cognitive Psychology, 7, 20-43.

COOPER, L. A. (1980). Spatial information processing: Strategies for research. In R. E. Snow, P.-A. Federico, \& W. E. Montague (Eds.), Aptitude, leaming and instruction: Vol. 1. Cognitive process analyses of aptitude. Hillsdale, NJ: Erlbaum.

COOPER, L. A., \&HEPARD, R. N. (1973). Chronometric studies of the rotation of mental images. In W. G. Chase (Ed.), Visual information processing. New York: Academic Press.

EgAN, D. E. (1978). Characterizing spatial ability: Different mental processes reflected in accuracy and latency scores (Research Rep. No. 1224). Pensacola, FL: Naval Aerospace Medical Research Laboratory.

EGAN, D. E. (1979). Testing based on understanding: Implications from studies of spatial ability. Intelligence, 3, 1-15.

Frfedericksen, J. R. (1980). Component skills in reading: Measurement of individual differences through chronometric analysis. In R. E. Snow, P.-A. Federico, \& W. E. Montague (Eds.), Aptitude, learning and instruction: Vol. 1. Cognitive process analyses of aptitude. Hillsdale, NJ: Erlbaum.

HUNT, E. (1980). The foundations of verbal comprehension. In R. E. Snow, P.-A. Federico, \& W. E. Montague (Eds.), Aptitude, learning and instruction: Vol. 1. Cognitive process analyses of aptitude. Hillsdale, NJ: Erlbaum.

Just, M. A., \& CARPENTER, P. A. (1976). Eye fixations and cognitive processes. Cognitive Psychology, 8, 441-480.

Kail, R., Pellegrino, J., \& CarTer, P. (1980). Developmental changes in mental rotation. Journal of Experimental Child Psychology, 29, 102-116.

LANSMAN, M. (1981). Ability factors and the speed of information processing. In M. P. Friedman, J. P. Das, \& N. O'Connor (Eds.), Intelligence and learning. New York: Plenum Press.

Lohman, D. F. (1979). Spatial ability: A review and reanalysis of the correlational literataure (Tech. Rep. No. 8). Stanford, CA: Stanford University, Aptitude Research Project, School of Education.

MCGeE, M. G. (1979). Human spatial abilities: Sources of sex differences. New York: Praeger.

METzLER, J., SHEPaRd, R. N. (1974). Transformational studies of the internal representations of three-dimensional objects. In $R$. Solso (Ed.), Theories in Cognitive Psychology: The Loyola Symposium. Potomac, MD: Erlbaum.

Pellegrino, J. W., \&laser, R. (1980). Components of inductive reasoning. In R. E. Snow, P.-A. Federico, \& W. E. Montague (Eds.), Aptitude, learning and instruction: Vol. 1. Cognitive process analyses of aptitude. Hillsdale, NJ: Erlbaum.

Pellegrino, J. W., \& Glaser, R. (1982). Analyzing aptitudes for learning: Inductive reasoning. In R. Glaser (Ed.), Advances in instructional psychology (Vol. 2). Hillsdale, NJ: Erlbaum. 
Pellegrino, J. W., \& KaIL, R. V. (1982). Process analyses of spatial aptitude. In R. J. Sternberg (Ed.), Advances in the psychology of human intelligence (Vol. 1). Hillsdale, NJ: Erlbaum.

Pellegrino, J. W., \& Mumaw, R. J. (1980). Multicomponent models of spatial ability. Unpublished manuscript, University of California at Santa Barbara, Santa Barbara, CA.

Pylyshyn, Z. W. (1979). The rate of "mental rotation" of images: A test of a holistic analogue hypothesis. Memory \& Cognition, 7 , 19-28.

SHEPARD, R. N. (1975). Form, formation, and transformation of internal representations. In R. L. Solso (Ed.), Information Processing and Cognition: The Loyola Symposium. Hillsdale, NJ: Erlbaum.

SMith, I. (1964). Spatial ability: Its educational and social significance. London: University of London Press.

STERNBERG, R. (1977). Intelligence, information processing and analogical reasoning. Hillsdale, $\mathrm{NJ}$ : Erlbaum.

STERNBERG, R. J. (1979). The nature of mental abilities. American Psychologist, 34, 214-230.

ThURSTONE, L. L. (1938). Primary mental abilities, Chicago: University of Chicago Press.

Thurstone, T. G. (1958). Manual for the SRA Primary Mental Abilities. Chicago: Science Research Associates.

\section{NOTES}

1. Values of $0^{\circ}-150^{\circ}$ were used because linear trends are strongest over this range of values. Deviations from linearity can occur when a value of $180^{\circ}$ is used, and this may be due to the use of depth-plane rotations to solve these items. Values above $180^{\circ}$ were not used because the linear trend is symmetrical to that observed for the $0^{\circ}-150^{\circ}$ range; that is, an angular disparity of $270^{\circ}$ is equivalent to a $90^{\circ}$ rotation (Cooper \& Shepard, 1973).

2. Two subjects from the total sample of 99 were eliminated in this and all subsequent analyses due to their aberrant negative scores on the PMA Space Test. Such scores indicate either unwillingness to respond appropriately to the test or misunderstanding of the test directions.

3. The data from different-judgment trials were not used for two reasons. First, 51 subjects had half as many different-judgment trials as same-judgment trials, and thus the data are less reliable for process estimates. Second, there are significant interpretive problems associated with using different-judgment slopes and intercepts. These interpretive problems involve individual differences in the model that captures different-trial processing. For a more complete discussion of this problem, see Pellegrino and Kail (1982).

4 . The reduced correlations between slope and intercept measures within a stimulus type may be partly due to artifactual negative correlations that exist between slope and intercept values estimated from the same data. This is particularly problematic when the linear fit is poor. However, linear fits for individual subjects were quite good, thus mitigating major contributions of such an artifact in the present data. In addition, the lowest correlation between slope and intercept measures was obtained for the relationship between the alphanumeric intercept and PMA slope, measures obtained from independent data sets.

(Manuscript received August 21, 1982; revision accepted for publication June 18, 1984.) 